\title{
MedienPädagogik
}

Zeitschrift für Theorie und Praxis der Medienbildung

\section{Editorial: Medien in der Erziehungswissenschaft II}

Werner Sesink

Im Juli 2004 fand in Darmstadt die zweite MEWISS-Tagung («Medien in der Erziehungswissenschaft») statt, organisiert vom Arbeitsbereich «Bildung und Technik» des Instituts für Allgemeine Pädagogik und Berufspädagogik der TU Darmstadt in Zusammenarbeit mit der Kommission Medienpädagogik der Deutschen Gesellschaft für Erziehungswissenschaft. Fortgesetzt wurde damit der 2003 am Duisburg Learning Lab der Universität Duisburg-Essen begonnene Erfahrungs- und Gedankenaustausch zum Einsatz computer- und netzwerkgestützter Medien in der erziehungswissenschaftlichen Hochschullehre. Damit wurde das Ziel verfolgt, die Diskussion in der erziehungswissenschaftlichen Community über Medienfragen im deutschsprachigen Raum anzuregen, den Austausch über entsprechende Aktivitäten zu fördern, zu einer Verbreitung verfügbarer Lösungen beizutragen, Anknüpfungspunkte für die gemeinsame Fortführung entsprechender Aktivitäten zu finden und vorliegende Kompetenzen zu vernetzen. Dem Tagungsziel entsprechend stellten die Autorinnen und Autoren der Beiträge sich insbesondere der Frage, worin der pädagogische Sinn des Einsatzes Neuer Medien in der Hochschullehre liegt und wie die zur Verfügung stehenden medialen Formen und Mittel für eine pädagogisch verantwortete Praxis zur Wirkung gebracht werden können.

Christian Swertz führt in das Konzept einer «Web-Didaktik» ein, indem er seinen Beitrag selbst webdidaktisch organisiert. Werner Sesink und Mitarbeiter zeigen an einem Beispiel aus dem Studienfach Pädagogik an der TU Darmstadt, dass und wie es möglich ist, eine Vorlesung durch Einsatz eines «interaktiven Skripts» so zu transformieren, dass das aktive und diskursive Arbeiten mit den Vorlesungsinhalten erfolgreich angeregt wird. Entlastung von «überflüssiger Präsenzlehre», um Kapazitäten freizusetzen für aktivierende Lehrformen, ist ein an der TU Kaiserslautern im Lehrbereich Berufs- und Wirtschaftspädagogik verfolgter Ansatz, von dem Markus Lermen berichtet. Eine «Lern-, Studier- und Forschungswerkstatt» für die Entwicklung eigener Projekte im Bereich innovativen Medieneinsatzes im Unterricht durch Studierende der Medieninformatik und der Lehrämter stellt die Arbeitsgruppe Hendrik Bunke, Sandra Ostermann, Elin-Birgit Berndt von der Universität Bremen vor.

Auch mit dieser Ausgabe verbindet sich die Hoffnung, weitere Impulse und Anregungen zum Medieneinsatz in der erziehungswissenschaftlichen Lehre geben zu können.

Sesink, Werner. 2006. «Editorial: Medien in der Erziehungswissenschaft Il». MedienPädagogik 10, (März), i. https://doi.org/10.21240/ mpaed/10/2006.03.29.X. 